\title{
BMJ Open Soluble urokinase plasminogen activator receptor (suPAR) as a prognostic marker of mortality in healthy, general and patient populations: protocol for a systematic review and meta-analysis
}

\author{
Jens Emil Vang Petersen, ${ }^{1}$ Thomas Kallemose,${ }^{2}$ Karen D Barton, ${ }^{3}$ \\ Avshalom Caspi, ${ }^{4,5,6,7}$ Line Jee Hartmann Rasmussen (i) ${ }^{2,4}$
}

To cite: Petersen JEV, Kallemose T, Barton KD, et al. Soluble urokinase plasminogen activator receptor (suPAR) as a prognostic marker of mortality in healthy, general and patient populations: protocol for a systematic review and meta-analysis. BMJ Open 2020;10:e036125. doi:10.1136/ bmjopen-2019-036125

- Prepublication history and additional material for this paper are available online. To view these files, please visit the journal online (http://dx.doi. org/10.1136/bmjopen-2019036125).

Received 01 December 2019 Revised 11 February 2020 Accepted 12 May 2020

Check for updates

(c) Author(s) (or their employer(s)) 2020. Re-use permitted under CC BY-NC. No commercial re-use. See rights and permissions. Published by BMJ.

For numbered affiliations see end of article.

\section{Correspondence to}

Dr Line Jee Hartmann Rasmussen;

line.jee.hartmann.rasmussen@ duke.edu

\section{ABSTRACT}

Introduction Chronic inflammation is increasingly recognised as a major contributor to disease, disability and ultimately death, but measuring the levels of chronic inflammation remains non-canonised, making it difficult to relate chronic inflammation and mortality. Soluble urokinase plasminogen activator receptor (suPAR), an emerging biomarker of chronic inflammation, has been proposed as a prognostic biomarker associated with future incidence of chronic disease and mortality in general as well as patient populations. Proper prognostic biomarkers are important as they can help improve risk stratification in clinical settings and provide guidance in treatment or lifestyle decisions as well as in the design of randomised trials. Here, we wish to summarise the evidence about the overall association of the biomarker suPAR with mortality in healthy, general and patient populations across diseases.

Methods and analysis The search will be conducted using Medline, Embase and Scopus databases from their inception to 03 June 2020 to identify studies investigating 'suPAR' and 'mortality'. Observational studies and control groups from intervention studies written in English or Danish will be included. The 'Quality In Prognosis Studies' tool will be used to assess the risk of bias for the studies included. Unadjusted and adjusted mortality outcome measures (eg, risk ratios, ORs, HRs) with $95 \%$ Cls will be extracted for healthy individuals, general and patient populations. The primary outcome is all-cause mortality within any given follow-up. Subgroup analyses will be performed based on time of outcome, cause of death, population type, adjustments for conventional risk factors and inflammation markers. Ethics and dissemination This systematic review will synthesise evidence on the use of suPAR as a prognostic marker for mortality. The results will be disseminated by publication in a peer-reviewed journal. Data used will be obtained from published studies, and ethics approval is therefore not necessary for this systematic review.

Trial registration number

PROSPERO CRD42020167401.
Strengths and limitations of this study

- To the best of our knowledge, this is the first systematic review and meta-analysis that investigates the association between soluble urokinase plasminogen activator receptor (suPAR) and mortality across general and patient populations.

- This review will provide valuable new knowledge for researchers studying chronic inflammation's effect on both short- and long-term health, and for clinicians using suPAR in clinical settings to stratify patients.

- Study selection, data extraction and quality assessment will be performed independently by two reviewers.

- The results will be discussed in context with other studies in the field.

- Common to most meta-analyses, significant heterogeneity may exist, which will be investigated thoroughly with subgroup analyses and meta-regressions.

\section{INTRODUCTION}

Rationale

Chronic inflammation is increasingly recognised as a major contributor to disease, disability and ultimately death in industrialised and low/middle-income countries alike. ${ }^{1-4}$ Chronic inflammation is related to multiple genetic and lifestyle factors, but measuring the levels of chronic inflammation remains non-canonised, making it difficult to relate chronic inflammation and death. Soluble urokinase plasminogen activator receptor (suPAR) is a protein present in the blood, and its concentration is thought to reflect a person's level of chronic inflammation and immune activation. ${ }^{56}$ Thus, elevated 
suPAR is proposed as a prognostic biomarker associated with future incidence of chronic disease and mortality in general as well as patient populations, ${ }^{78}$ including previous systematic reviews and meta-analyses showing suPAR to be elevated in focal segmental glomerulosclerosis ${ }^{910}$ or to be associated with mortality in patients with bacterial infections and sepsis. ${ }^{11-14}$ While healthy persons generally have a low level of suPAR in the blood, ${ }^{15}$ the blood concentration of suPAR is increased in a wide range of diseases: acute and chronic, non-communicable and infectious, that is, suPAR has been shown to be elevated in cardiovascular diseases (stroke, ischaemic heart disease, venous thromboembolism, incident atrial fibrillation), ${ }^{16-18}$ type 1 and type 2 diabetes, ${ }^{19-21}$ various types of cancer, ${ }^{22-36}$ rheumatic disease, ${ }^{37}$ chronic pulmonary disease, ${ }^{39}$ chronic liver disease (non-alcoholic fatty liver disease, cirrhosis) ${ }^{40-42}$ chronic kidney disease ${ }^{4344}$ as well as infectious diseases caused by viruses, ${ }^{42}{ }^{45-47}$ bacteria ${ }^{48-57}$ and parasites. ${ }^{58} 59$ Together, these studies highlight the broad associations across patient groups and aetiologies-and even in general populations-between elevated blood levels of suPAR with general health, disease outcome, complications and mortality.

In contrast to common inflammatory biomarkers, such as the current gold-standard C-reactive protein (CRP), suPAR is not an acute-phase reactant, and suPAR levels in the blood are less rapidly affected by acute changes and short-term influences. ${ }^{17} 60$ Additionally, suPAR was more reliably associated with early-life risk factors such as adverse childhood experiences, early-life stress and violence than CRP and interleukin-6 (IL-6), potentially because these more traditional biomarkers of inflammation as acute-phase reactants mix historical and acute effects. ${ }^{6162}$ This, along with its non-specific associations with pathologies in general, suggests that suPAR blood levels are an appropriate readout for chronic inflammation.

Prognostic biomarkers are important as they can help improve risk stratification in clinical settings or provide guidance in treatment or lifestyle decisions as well as in the design of randomised trials. ${ }^{63}$ Here, we wish to summarise the evidence about the overall association of the biomarker suPAR with mortality in healthy, general and patient populations and across diseases. As suPAR is still a relatively new clinical biomarker, clinical guidelines and cut-offs are still lacking. Our findings will clarify the association between suPAR and mortality, and what value a biomarker reflecting chronic inflammation adds, compared with the current standard inflammatory biomarkers. The study will help development of future clinical guidelines, based on a better understanding of differences in the prognostic value of suPAR between and across healthy individuals and patient subgroups, which is critical in clinical decision making. Having an established accurate chronic inflammation biomarker with a well-described association with mortality is a vital tool in future efforts to combat major public health challenges.

\section{Objective}

In this systematic review, we aim to investigate the hypothesis that elevated suPAR is associated with increased risk of short-term and long-term mortality in healthy, general and patient populations, independent of conventional risk factors.

To this end, the proposed systematic review will answer the following questions:

Primary aim:

1. Do individuals with higher suPAR levels have a higher risk of mortality?

Secondary aims:

1. Is the association between suPAR and mortality present in healthy, general and various patient populations?

2. Is the association between suPAR and mortality independent of conventional risk factors, such as age, sex, smoking and chronic disease?

3. Is the association between suPAR and mortality independent of other inflammatory biomarkers?

4. What is the discrimination performance of suPAR for predicting mortality?

5. What clinical and study methodological characteristics explain heterogeneity in the results?

\section{METHODS AND ANALYSIS \\ Review design}

The study protocol for this systematic review and metaanalysis was developed based on the Preferred Reporting Items for Systematic Reviews and Meta-Analyses Protocols (PRISMA-P) guidelines ${ }^{64} 65$ and was registered with PROSPERO (registration number CRD42020167401).

This study will follow the recommendations on conducting and reporting systematic reviews and metaanalyses set forth by the PRISMA ${ }^{66}$ and Meta-analysis of Observational Studies in Epidemiology (MOOSE) ${ }^{67}$ guidelines, as well as the updated CHecklist for critical Appraisal and data extraction for systematic Reviews of prediction Modelling Studies (CHARMS) checklist for prognostic factors CHARMS- $(\mathrm{PF}) .{ }^{63}$

\section{Eligibility criteria}

Studies on suPAR and mortality will be selected according to the criteria outlined below.

\section{Study designs}

We will include prospective or retrospective observational studies (cohorts, case-control studies, nested casecontrol studies) and control groups from intervention studies. We will exclude animal experiments.

\section{Participants}

We will include studies examining healthy human individuals, general human populations or any human patient population. We will include studies of both children and adults without restrictions on ethnicity, sex or disease status. 
Index prognostic factor

We will include studies with suPAR measured in plasma or serum, independent of assay type, manufacturer or sample storage time and conditions (whether suPAR was measured in fresh or frozen samples); this information will be collected for quality assessment and heterogeneity analysis (described below in detail). We will exclude studies where suPAR was not measured in blood (eg, urine samples).

\section{Comparators}

We will investigate the unadjusted and adjusted prognostic value of suPAR, that is, without and with adjustments for other PFs, for example, conventional risk factors (such as age, sex, smoking and chronic disease), inflammatory biomarkers (such as CRP, white blood cells and IL-6), or kidney function (such as creatinine and glomerular filtration rate).

\section{Outcomes}

We will investigate the outcome of mortality. We will include studies with outcomes reported as unadjusted or adjusted effect estimates of relative risk (eg, risk ratio (RR), OR, HR). In studies reporting mortality as part of a composite outcome measure, we will extract all individual outcomes as reported in the studies. We will extract the outcome in all data forms (for example, dichotomous-30 days mortality yes/no; continuous-time to death) as reported in the included studies. For studies reporting survival from time-to-event analyses, we will use this information to extract the number of deaths. Further, we will investigate the discriminative ability of suPAR as a secondary outcome, that is, area under the curves (AUCs) for receiver operating characteristics (ROC) curve analyses of suPAR and mortality. We will exclude studies of deaths due to external/unnatural causes, such as homicide, suicides, accidents, drug overdoses and medical errors.

\section{Timing}

We will investigate the association between suPAR and mortality during any given period of follow-up. We will exclude cross-sectional studies.

\section{Setting}

There will be no restrictions by type of setting.

\section{Language and publication type}

We will include peer-reviewed studies in English or Danish published through 03 June 2020. We will exclude reviews, commentaries, correspondence, case reports, conference abstracts, expert opinions, editorials, experimental studies and dissertations. A list of possibly relevant titles in other languages will be provided as an online supplementary appendix.

\section{Information sources}

The following databases will be searched from their inception forward for potentially eligible studies published on or before 03 June 2020: (1) Medline via PubMed, (2) Embase via Elsevier and (3) Scopus via Elsevier. The electronic database search will be supplemented with a hand search of reference lists of included studies. Finally, we will circulate a bibliography of the included articles to the systematic review team, as well as to suPAR experts identified by the team. The electronic databases search will be carried out by KDB (Biomedical Research Liaison Librarian), and the supplemental hand search will be carried out by JEVP and LJHR.

\section{Search strategy}

The specific search strategy was created by a Biomedical Research Liaison Librarian (KDB) with expertise in systematic review searching. The search strategy was developed with input from the project team. The search uses medical subject headings terms and keywords related to suPAR and mortality. No study design, date or language limits will be imposed on the search. The following terms will be used to search the electronic databases in addition to other related terms for the concepts of 'suPAR' and 'mortality':

"suPAR" or "soluble urokinase plasminogen activator receptor" or "soluble urokinase-type" or "soluble urokinase receptor" or "uPAR"

\section{AND}

"mortality" or "death" or "fatality".

The initial search will be performed on 03 June 2020. Searches will be repeated prior to publication. The full PubMed search and search terms are shown in online supplementary appendix 1.

\section{Study records}

Data management

Citations extracted from electronic databases will by imported to EndNote. The Covidence systematic review software will be used for the screening and review processes, including removal of duplicates. For the actual data extraction, a data codebook will be a priori developed in Microsoft Excel based on a pilot search, along with a manual describing the information to be entered under each data item in the codebook.

\section{Selection process}

Two reviewers (JEVP and LJHR) will independently screen titles and abstracts yielded by the search to identify eligible studies according to the inclusion criteria. Studies that do not meet the screening criteria will be excluded. We will obtain full reports for all titles that appear to meet the inclusion criteria or where there is any uncertainty. The same two reviewers (JEVP and LJHR) will independently review the full-text articles to assess for eligibility. The included and excluded studies will be checked and reasons for inclusion/exclusion will be verified. Disagreements will be resolved by consensus, or by a third author if necessary. Reasons for exclusion will be coded for both the initial screening and for the review of the full-text articles. The PRISMA flow diagram 
will be used to document the study selection process. An appendix with a reference list of all excluded studies will be included in the final manuscript. Neither of the reviewers will be blind to the article titles, study authors, or institutions. Multiple reports of a single study will be identified by juxtaposing author names, study names, institutions, study dates. To avoid double counting, in cases of duplicate publications or multiple reports from the same study that all meet the inclusion criteria, the reviewers will select publications based on the following prioritisation: reports with (1) adjusted analyses; (2) more covariates included; (3) bigger sample size. In cases where different reports from the same study provide unique data on different follow-up times, adjustments or subgroups, unique information from the individual reports will be extracted for the main analysis, subgroup analyses and meta-regressions.

\section{Data collection process}

Data will be extracted from reports and entered in the Excel codebook in duplicate by the two independent reviewers (JEVP and LJHR). As mentioned, the data extraction codebook is developed a priori with statistical consultancy from TK. To ensure consistency across reviewers, we will conduct calibration exercises before starting the data extraction. The extracted data will include all the necessary information to describe and characterise the studies, assess the quality, synthesise data for the meta-analyses and to assess heterogeneity. In case of missing data or insufficient reporting of details, the study's corresponding author will be contacted for clarification, if possible, by a maximum of three email attempts. When data extraction is completed, both authors will review the codebooks and resolve any discrepancies by consensus or by a third author if necessary. Prior to correcting disagreements, the overall inter-rater agreement rate will be calculated using Cohen's $\kappa$ statistic ( $>0.80$ is considered good). A list of extracted variables will be provided as an appendix in the final manuscript. For studies consisting of multiple groups of individuals (eg, healthy controls, patients with precancerous lesions and patients with cancer), individual group information will be extracted to assess the association between suPAR and mortality for each group.

\section{Data items}

The major categories of extracted data will be: (1) study characteristics (author, journal, year of publication, country/region, funding sources, etc); (2) study design (type of study, year of study start, duration of follow-up, etc); (3) study population (sample size at baseline, population characteristics (healthy individuals, general population, patient types), age, sex, sample size at follow-up, reasons for loss to follow-up, information about treatments, etc), (4) index suPAR (suPAR levels, distribution, assay type, manufacturer, comparison groups and cutoffs, etc); (5) outcomes (including mortality/survival rates; cause of death; suPAR levels stratified by survivors/ non-survivors; unadjusted, minimally adjusted and most adjusted RR, OR and/or HR for short-term and longterm all-cause mortality; and true positive, false positive (FP), true negative, and false negative frequencies as well as AUCs for ROC curves); (6) control characteristics (conventional risk factors, eg, age, sex, smoking and chronic diseases; other inflammatory biomarkers, eg, CRP, white blood cell count, cytokines and fibrinogen; and kidney function, eg, creatinine (measured or estimated), creatinine clearance, glomerular filtration rate (measured or estimated)); (7) setting (general population, healthcare setting, eg, acute care, intensive care unit, outpatients, etc).

\section{Outcomes and prioritisation}

The primary outcome is all-cause mortality within any given follow-up period. Reports that are not indicating cause of deaths will be analysed under all-cause mortality.

When studies report mortality/survival rates at various time points of the follow-up, we have decided a priori to subdivide the mortality rates as follows:

1. Short-term mortality: Death within 30 days from baseline.

2. 30-365 days mortality: Death occurring between 30 days and 365 days from baseline.

3. Long-term mortality: Death occurring more than 365 days from baseline.

For the primary meta-analysis, the most long-term outcome will be used, that is, if a study reports associations between suPAR and mortality at multiple time points, the more long-term assessment of mortality will be used. Furthermore, we will conduct subgroup analyses stratifying studies reporting mortality within 30 days, between 30 and 365 days and more than 365 days, as described in detail in the 'Subgroup analyses and meta-regression' section.

Secondary outcomes will be:

1. Short-term mortality (within 30 days) of any cause (allcause mortality).

2. Cardiovascular mortality.

3. Cancer mortality.

4. Discriminative ability of suPAR, that is, AUCs for ROC curves of suPAR and mortality for the most long-term outcome reported.

\section{Risk of bias in individual studies (quality assessment)}

To facilitate the assessment of possible risk of bias, the methodological quality of each study will be evaluated using the Quality in Prognosis Studies (QUIPS) tool, table $1 .^{68}$ The QUIPS tool assesses risk of bias across six domains in studies of PFs: (1) study participation (sampling bias); (2) study attrition (attrition bias); (3) PF measurement; (4) outcome measurement; (5) study confounding; and (6) statistical analysis and reporting. The QUIPS tool will be adapted to meet the specific needs of this systematic review. To ensure consistency across reviewers, we will conduct calibration exercises before starting the quality assessments. Neither of the reviewers will be blinded to 
Table 1 The Hayden, Côté and Bombardier QUIPS risk of bias assessment instrument for prognostic factor (PF) studies

Biases

Instructions to assess the risk of each potential bias:

\section{Study participation}

Source of target population

Method used to identify population

Recruitment period

Place of recruitment

Inclusion and exclusion criteria

Adequate study participation

Baseline characteristics

Study participation summary

\section{Study attrition}

Proportion of baseline sample available for analysis

Attempts to collect information on participants who dropped out

Reasons and potential impact of subjects lost to follow-up

Outcome and PF information on those lost to follow-up

Study attrition summary

\section{PF measurement}

Definition of the PF

Valid and reliable measurement of PF

Method and setting of PF measurement The method and setting of measurement of PF is the same for all study participants.

Proportion of data on PF available for Adequate proportion of the study sample has complete data for PF variable. analysis

Method used for missing data

PF measurement summary

\section{Outcome measurement}

Definition of the outcome
Appropriate methods of imputation are used for missing 'PF' data.

\section{Issues to consider for judging overall rating of 'risk of bias'}

These issues will guide your thinking and judgement about the overall risk of bias within each of the six domains. Some 'issues' may not be relevant to the specific study or the review research question. These issues are taken together to inform the overall judgement of potential bias for each of the six domains.

Goal: To judge the risk of selection bias (likelihood that relationship between PF and outcome is different for participants and eligible non-participants).

The source population or population of interest is adequately described for key characteristics.

The sampling frame and recruitment are adequately described, including methods to identify the sample sufficient to limit potential bias (number and type used, eg, referral patterns in healthcare).

Period of recruitment is adequately described.

Place of recruitment (setting and geographical location) are adequately described.

Inclusion and exclusion criteria are adequately described (eg, including explicit diagnostic criteria or 'zero time' description).

There is adequate participation in the study by eligible individuals.

The baseline study sample (ie, individuals entering the study) is adequately described for key characteristics.

The study sample represents the population of interest on key characteristics, sufficient to limit potential bias of the observed relationship between PF and outcome.

Goal: To judge the risk of attrition bias (likelihood that relationship between PF and outcome are different for completing and non-completing participants).

Response rate (ie, proportion of study sample completing the study and providing outcome data) is adequate.

Attempts to collect information on participants who dropped out of the study are described.

Reasons for loss to follow-up are provided.

Participants lost to follow-up are adequately described for key characteristics.

There are no important differences between key characteristics and outcomes in participants who completed the study and those who did not.

Loss to follow-up (from baseline sample to study population analysed) is not associated with key characteristics (ie, the study data adequately represent the sample) sufficient to limit potential bias to the observed relationship between PF and outcome.

Goal: To judge the risk of measurement bias related to how PF was measured (differential measurement of PF related to the level of outcome).

A clear definition or description of 'PF' is provided (eg, including dose, level, duration of exposure and clear specification of the method of measurement).

Method of PF measurement is adequately valid and reliable to limit misclassification bias (eg, may include relevant outside sources of information on measurement properties, also characteristics, such as blind measurement and limited reliance on recall).

Continuous variables are reported or appropriate cut-points (ie, not data-dependent) are used.

$\mathrm{PF}$ is adequately measured in study participants to sufficiently limit potential bias.

Goal: To judge the risk of bias related to the measurement of outcome (differential measurement of outcome related to the baseline level of PF).

A clear definition of outcome is provided, including duration of follow-up and level and extent of the outcome construct. 
Table 1 Continued

Biases Issues to consider for judging overall rating of 'risk of bias'

Valid and reliable measurement of outcome

The method of outcome measurement used is adequately valid and reliable to limit misclassification bias (eg, may include relevant outside sources of information on measurement properties, also characteristics, such as blind measurement and confirmation of outcome with valid and reliable test).

\section{Method and setting of outcome} measurement

Outcome measurement summary

\section{Study confounding}

Important confounders measured

Definition of the confounding factor

Valid and reliable measurement of confounders

The method and setting of outcome measurement is the same for all study participants.

Outcome of interest is adequately measured in study participants to sufficiently limit potentia bias.

Goal: To judge the risk of bias due to confounding (ie, the effect of PF is distorted by another factor that is related to PF and outcome).

All important confounders, including treatments (key variables in conceptual model), are measured.

Clear definitions of the important confounders measured are provided (eg, including dose, level and duration of exposures).

Measurement of all important confounders is adequately valid and reliable (eg, may include relevant outside sources of information on measurement properties, also characteristics, such as blind measurement and limited reliance on recall).

$\begin{array}{ll}\begin{array}{l}\text { Method and setting of confounding } \\ \text { measurement }\end{array} & \begin{array}{l}\text { The method and setting of confounding measurement are the same for all study participants. } \\ \text { Method used for missing data }\end{array} \\ \begin{array}{ll}\text { Appropriate accounting for confounding } \\ \text { Appopriate methods are used if imputation is used for missing confounder data. } \\ \text { variables, stratification, or initial assembly of comparable groups). } \\ \text { Important potential confounders are accounted for in the analysis (ie, appropriate } \\ \text { adjustment). }\end{array} \\ \begin{array}{ll}\text { Important potential confounders are appropriately accounted for, limiting potential bias with } \\ \text { respect to the relationship between PF and outcome. }\end{array} \\ \begin{array}{ll}\text { 6. Statistical analysis and reporting } \\ \text { Goal: To judge the risk of bias related to the statistical analysis and presentation of } \\ \text { results. }\end{array} \\ \begin{array}{l}\text { There is sufficient presentation of data to assess the adequacy of the analysis. } \\ \text { Model development strategy }\end{array} & \begin{array}{l}\text { The strategy for model building (ie, inclusion of variables in the statistical model) is } \\ \text { appropriate and is based on a conceptual framework or model. }\end{array} \\ \text { The selected statistical model is adequate for the design of the study. }\end{array}$

Modified from: Hayden JA, Côté P, Bombardier C. Evaluation of the quality of prognosis studies in systematic reviews. Ann Intern Med. 2006;144:427-37.

QUIPS, Quality in Prognosis Studies.

studies during the quality assessment. For each domain in the tool, we will describe the procedures undertaken for each study, including verbatim quotes. If there is insufficient detail reported in the study, we will judge the risk of bias as 'unclear' and the study's authors will be contacted for more information. Studies will be considered to have a low, moderate or high risk of bias according to the following scores of low risk across domains: 5-6, 3-4, 0-2. The two reviewers (JEVP and LJHR) will assess the risk of bias independent of each other. Any disagreements will be resolved by consensus, or if necessary by a third author, and a log of these will be included as an appendix in the final manuscript. No study will be excluded based on the results of risk of bias assessment. We will compute graphic representations of potential bias for the final manuscript. In the meta-analysis, subgroup analyses will be performed based on the risk of bias (QUIPS; low, moderate or high risk of bias). The adapted QUIPS tool will be provided as an appendix in the final manuscript along with the log of disagreements.

\section{Data synthesis}

Reported relative risks and their corresponding 95\%-99\% CIs will be used to assess the association between suPAR and most long-term mortality with random-effects meta-analyses to minimise between-study heterogeneity. A quantitative synthesis will be performed, and our outcomes will be studied separately in three pooled datasets: (1) 
across all studies (despite a high degree of expected heterogeneity), (2) within studies of healthy/general populations and (3) within studies of patient populations.

Relative risks with 95\%-99\% CIs will be used as the common measure of association across studies. RRs, ORs and HRs will be assumed to approximate the same measure of relative risk. As previously described for CRP and albumin, ${ }^{69} 70$ we will convert the reported studyspecific relative risk estimates for suPAR onto a standardised scale of effect, comparing the highest third with the lowest third of the suPAR distribution, that is, providing an estimate per 2.18 times SD units of suPAR. 2.18 is the difference in the means of the top and bottom third of the standard normal distribution and is therefore used as the point estimate for the lower and upper third of the suPAR distribution when scaled with SD. This method assumes that suPAR follows a normal distribution, or a transformation of suPAR, such as the logarithm, follows a normal distribution. Additionally, it is assumed that the suPAR SD estimates within the studies are similar when scaling; if this is not the case additional adjustment to account for this will be done and differences between calculation methods will be reported. If we conclude that these assumptions cannot be made for the studies, separate relative risk estimates (per suPAR unit, $\log 2$ (suPAR), Q1 vs Q4 suPAR, etc) analyses will be made instead of the standardised scale analysis.

For the primary analysis all study outcome measures (eg, $\mathrm{RR}, \mathrm{OR}$ and HR) will be pooled as a single measure, and all available studies will be included, regardless of population. If a study has multiple versions of the same model with different adjustments, the model with most adjustments will be included. In addition, we will conduct separate subgroup analyses, as described below, to account for the heterogeneity across methods of reporting outcomes and variation in adjustments made.

As suggested by Riley $e t a l,{ }^{63}$ in addition to the main analysis, we will conduct multiple meta-analyses separately based on the most long-term outcome stratified on the following levels: (1) population level: all data, healthy/ general populations and patients; (2) model adjustment: unadjusted, minimally adjusted (age and sex), adjusted for some conventional risk factors (eg, age, sex, chronic disease/Charlson score, smoking) or inflammatory markers (eg, CRP, cytokines, fibrinogen) and maximally adjusted (most adjusted estimate from each study); (3) outcome measure: RR, OR and HR.

Statistical heterogeneity among studies will be evaluated using the $\tau^{2}$ and $\mathrm{I}^{2}$ statistic (where $\mathrm{I}^{2}$ of $30 \%-60 \%$ will be interpreted to indicate moderate heterogeneity and $\mathrm{I}^{2}>50 \%$ to indicate substantial heterogeneity across studies). ${ }^{71}$ We will try to explain the source of heterogeneity by subgroup analysis or sensitivity analysis (see below).

Study characteristics of the included studies will be summarised in a table. To visually assess between-study variability, we will present the results and summary relative risks in forest plots.
Analysis of the predictive value of suPAR for mortality will be done by hierarchal summary ROC (HSROC) model curves. From this, SROC curves with AUCs, Qs and diagnostic ORs will be produced.

As described for CRP by Hemingway et $a l l^{69}$ we will attempt to calculate the detection rate (sensitivity) at different FP rates from 0 to 100 by constructing the lognormal distributions of suPAR separately for those who survived and those who died. From this we will obtain a ROC curve and report the c-statistic. Pooled estimates of both the c-statistic and detection rate of suPAR's discriminative ability for predicting mortality will be obtained by random-effects meta-analysis of the study-specific c-statistics and detection rates. CIs and a 10\% FP rate will be reported.

All statistical analyses will be performed using SAS Enterprise Guide 7.1 (SAS Institute Inc., Cary, NC, USA) and R (R Foundation for Statistical Computing, Vienna, Austria) software.

\section{Subgroup analyses and meta-regression}

In addition to the primary analysis of the most long-term mortality, separate analyses will be made for the following mortality outcomes: mortality within 30 days, 30-365 days, and long-term mortality (more than 365 days). These analyses will be done as described for the primary analysis above.

Subgroup analyses will be used to explore possible sources of heterogeneity, and univariate randomeffects meta-regression will be performed based on the following: study design (cohort, case-control, randomised controlled trials); year of study start; sex; age groups; time of outcome (within 30 days, 30-365 days, more than 365 days); reported relative risk estimates (eg, RR, OR, HR); population type (healthy/general population vs patient types, eg, cardiovascular disease, cancer, chronic kidney disease, infectious disease, critical illness, acute care); cause of death studied (all-cause, cardiovascular, cancer mortality, etc); methods of suPAR measurement; suPAR assay manufacturer; suPAR comparison group (continuous suPAR, equal sized groups, unequal sized groups); region (North America + Europe, Asia, Africa, South America); duration of follow-up; no. of adjustments; adjustment for CRP; adjustment for kidney function; no. of events; risk of bias (QUIPS; low, moderate, high risk of bias).

To explore other potential sources of heterogeneity, a random-effects meta-regression model will be employed, which includes study level continuous or categorical covariates.

\section{Sensitivity analysis}

Sensitivity analyses will be performed in which the pooled risk estimates are recalculated by removing the studies one by one and comparing the results. Furthermore, a sensitivity analysis of risk of bias will be performed by omitting studies that are judged to be at high risk of bias. 


\section{Meta-biases}

Small study bias (including publication bias) will be assessed with contour-enhanced Funnel plots, by Begg'sadjusted rank correlation test and by Egger's regression asymmetry test.

\section{Confidence in cumulative evidence}

Reporting and interpretation of results will follow the reporting guidelines of PRISMA ${ }^{66}$ and MOOSE. ${ }^{67}$ Interpretation and translation of summary results will follow these guidelines as well as the steps recommended for PF studies by Riley et $a .^{63}$ The summary results will be discussed in terms of potential usefulness for clinical practice and need for future research.

Strength in the body of evidence will be further evaluated using the Grades of Recommendation, Assessment, Development and Evaluation (GRADE) assessment. ${ }^{72} 73$ However, this approach was developed for the assessment of intervention effectiveness in reviews of interventions and not for assessing the certainty of summary results of systematic reviews of PFs; allowing for heterogeneity in the latter case may be more acceptable. ${ }^{63}$

\section{Patient and public involvement}

No patients involved.

\section{DISCUSSION}

The biomarker suPAR has been suggested to be a prognostic biomarker in the general population and various patient populations. However, clinical guidelines and cutoffs are still lacking, hampering the wide clinical utilisation of suPAR. Our findings in this systematic review and meta-analysis will clarify the association between suPAR and mortality, and establish its prognostic value across healthy and ill individuals, providing support for development of future clinical guidelines. Thus, we will discuss the usefulness of suPAR in clinical practice, in particular settings, or as a general marker of prognosis across populations.

Only few randomised studies have investigated the value of adding suPAR as a prognostic biomarker to inform clinical practice, ${ }^{74}$ and most evidence is based on observational studies of suPAR, but many studies have reported an association between suPAR and mortality. Summarising this evidence is important to establish the prognostic role of suPAR. This protocol has been developed in compliance with recommended guidelines for PF studies, ${ }^{63}$ including PRISMA-P, ${ }^{64}$ and it provides a clear and structured protocol for maximising data extraction and summarising the relevant information on the importance of suPAR as a prognostic marker of mortality. suPAR is used as a marker of inflammation, and as such, many studies have compared it with CRP, although suPAR has been suggested to be a marker of chronic rather than acute inflammation while CRP is an acute phase reactant and potentially reflects a distinct aspect of inflammation. In adjusted analyses, suPAR has been shown to be associated with mortality independent of CRP. ${ }^{876}$ In our analyses, we aim to investigate the associations between suPAR and mortality in studies adjusting for CRP to assess the effect over and above CRP. The advantage of using a chronic inflammation marker rather than an acute phase reactant for prognostication includes the lower variation and sensitivity towards acute, short-term influences and a better assessment of underlying health status.

Blood suPAR levels have been associated with kidney function $^{77}$ and proposed a causal factor of certain chronic kidney diseases. ${ }^{78}$ The potential causal effect in kidney disease is outside the scope of this review. However, we will investigate whether suPAR is associated with mortality in individuals with and without chronic kidney disease.

Our primary aim of summarising all evidence of suPAR and mortality in one meta-analysis imposes a high degree of study population heterogeneity on this study; however, to establish an association between suPAR and mortality, it is important to summarise the information available on this issue and it will provide us with a general estimate of association. We will account for the heterogeneity by performing meta-regressions and stratified analyses to investigate the association in more homogeneous subsets of the literature.

This systematic review and meta-analysis will provide an up-to-date global overview of the current literature on suPAR and mortality. If our results indicate an association between suPAR level and mortality risk, suPAR may constitute an easily measurable, accurate chronic inflammation biomarker with a well-described association with mortality, which could be a vital tool in future efforts to combat major public health challenges, such as chronic disease prevention and premature mortality, and improve future research on this topic.

\section{ETHICS AND DISSEMINATION}

This systematic review will synthesise evidence on the use of suPAR as a prognostic marker for mortality based on published publicly available studies and data. The study will not obtain, store or report any individual-level personal information and there will be no concerns about privacy. Therefore, ethical approval is not necessary for this systematic review. The results will be disseminated by publication in a peer-reviewed journal.

\section{Author affiliations}

${ }^{1}$ Division of Infectious Diseases, Duke University School of Medicine, Durham, North Carolina, USA

${ }^{2}$ Department of Clinical Research, Copenhagen University Hospital Amager and Hvidovre, Hvidovre, Denmark

${ }^{3}$ Duke University Medical Center Library \& Archives, Duke University, Durham, North Carolina, USA

${ }^{4}$ Department of Psychology and Neuroscience, Duke University, Durham, North Carolina, USA

${ }^{5}$ Department of Psychiatry and Behavioral Sciences, Duke University School of Medicine, Durham, North Carolina, USA

${ }^{6}$ Center for Genomic and Computational Biology, Duke University, Durham, North Carolina, USA 
${ }^{7}$ Social, Genetic, and Developmental Psychiatry Centre, Institute of Psychiatry, Psychology, and Neuroscience, King's College London, London, UK

Contributors JEVP and LJHR are the guarantors of the review. All authors (JEVP, TK, KDB, AC and LJHR) contributed to the development of the selection criteria, the risk of bias assessment strategy and data extraction criteria. LJHR and JEVP conceived the study. JEVP, KDB and LJHR developed the search strategy. TK provided statistical expertise. JEVP and LJHR drafted the protocol. All authors (JEVP, TK, KDB, AC and LJHR) read, provided feedback and approved the final protocol.

Funding This research received no specific grant from any funding agency in the public, commercial, or not-for-profit sectors. JEVP is supported by an international postdoc fellowship from the Alfred Benzon Foundation (grant no. ABF-2018-91). LJHR is supported by an international postdoc fellowship from the Lundbeck Foundation (grant no. R288-2018-380).

Competing interests LJHR has received funding for travel outside the submitted work from ViroGates A/S.

Patient consent for publication Not required.

Provenance and peer review Not commissioned; externally peer reviewed.

Open access This is an open access article distributed in accordance with the Creative Commons Attribution Non Commercial (CC BY-NC 4.0) license, which permits others to distribute, remix, adapt, build upon this work non-commercially, and license their derivative works on different terms, provided the original work is properly cited, appropriate credit is given, any changes made indicated, and the use is non-commercial. See: http://creativecommons.org/licenses/by-nc/4.0/.

ORCID iD

Line Jee Hartmann Rasmussen http://orcid.org/0000-0001-6613-2469

\section{REFERENCES}

1 Hunter $\mathrm{P}$. The inflammation theory of disease. The growing realization that chronic inflammation is crucial in many diseases opens new avenues for treatment. EMBO Rep 2012;13:968-70.

2 Medzhitov R. Inflammation 2010: new adventures of an old flame. Cell 2010;140:771-6.

3 Franceschi C, Campisi J. Chronic inflammation (inflammaging) and its potential contribution to age-associated diseases. J Gerontol A Biol Sci Med Sci 2014;69:S4-9.

4 Michaud M, Balardy L, Moulis G, et al. Proinflammatory cytokines, aging, and age-related diseases. J Am Med Dir Assoc 2013;14:877-82.

5 Thunø M, Macho B, Eugen-Olsen J. suPAR: the molecular crystal ball. Dis Markers 2009;27:157-72.

6 Desmedt S, Desmedt V, Delanghe JR, et al. The intriguing role of soluble urokinase receptor in inflammatory diseases. Crit Rev Clin Lab Sci 2017;54:117-33.

7 Eugen-Olsen J, Andersen O, Linneberg A, et al. Circulating soluble urokinase plasminogen activator receptor predicts cancer, cardiovascular disease, diabetes and mortality in the general population. J Intern Med 2010;268:296-308.

8 Rasmussen LJH, Ladelund S, Haupt TH, et al. Soluble urokinase plasminogen activator receptor (suPAR) in acute care: a strong marker of disease presence and severity, readmission and mortality. A retrospective cohort study. Emerg Med J 2016;33:769-75.

9 Shuai T, Pei Jing Y, Huang Q, et al. Serum soluble urokinase type plasminogen activated receptor and focal segmental glomerulosclerosis: a systematic review and meta-analysis. BMJ Open 2019;9:e031812.

10 Lee JM, Yang JW, Kronbichler A, et al. Increased serum soluble urokinase-type plasminogen activator receptor (suPAR) levels in FSGS: a meta-analysis. J Immunol Res 2019;2019:1-11.

11 Pregernig A, Müller M, Held U, et al. Prediction of mortality in adult patients with sepsis using six biomarkers: a systematic review and meta-analysis. Ann Intensive Care 2019;9:125.

12 Backes Y, van der Sluijs KF, Mackie DP, et al. Usefulness of suPAR as a biological marker in patients with systemic inflammation or infection: a systematic review. Intensive Care Med 2012;38:1418-28.

13 Ni W, Han Y, Zhao J, et al. Serum soluble urokinase-type plasminogen activator receptor as a biological marker of bacterial infection in adults: a systematic review and meta-analysis. Sci Rep 2016;6:39481.

14 Huang $\mathrm{Q}$, Xiong $\mathrm{H}$, Yan $\mathrm{P}$, et al. The diagnostic and prognostic value of Supar in patients with sepsis: a systematic review and metaanalysis. Shock 2020;53:53:416-25.
15 Haastrup E, Grau K, Eugen-Olsen J, et al. Soluble urokinase plasminogen activator receptor as a marker for use of antidepressants. PLoS One 2014;9:e110555.

16 Persson M, Östling G, Smith G, et al. Soluble urokinase plasminogen activator receptor: a risk factor for carotid plaque, stroke, and coronary artery disease. Stroke 2014;45:18-23.

17 Lyngbæk S, Marott JL, Møller DV, et al. Usefulness of soluble urokinase plasminogen activator receptor to predict repeat myocardial infarction and mortality in patients with ST-segment elevation myocardial infarction undergoing primary percutaneous intervention. Am J Cardiol 2012;110:1756-63.

18 Westin O, Rasmussen LJH, Andersen O, et al. Soluble urokinase plasminogen activator receptor (suPAR) as a predictor of incident atrial fibrillation. J Atr Fibrillation 2018;10:1801.

19 Theilade S, Lyngbaek S, Hansen TW, et al. Soluble urokinase plasminogen activator receptor levels are elevated and associated with complications in patients with type 1 diabetes. J Intern Med 2015;277:362-71.

20 Heraclides A, Jensen TM, Rasmussen SS, et al. The proinflammatory biomarker soluble urokinase plasminogen activator receptor (suPAR) is associated with incident type 2 diabetes among overweight but not obese individuals with impaired glucose regulation: effect modification by smoking and body weight status. Diabetologia 2013;56:1542-6.

21 Guthoff M, Wagner R, Randrianarisoa E, et al. Soluble urokinase receptor (suPAR) predicts microalbuminuria in patients at risk for type 2 diabetes mellitus. Sci Rep 2017;7:40627.

22 Mustjoki S, Sidenius N, Sier CF, et al. Soluble urokinase receptor levels correlate with number of circulating tumor cells in acute myeloid leukemia and decrease rapidly during chemotherapy. Cancer Res 2000;60:7126-32.

23 Mustjoki S, Alitalo R, Stephens RW, et al. Blast cell-surface and plasma soluble urokinase receptor in acute leukemia patients: relationship to classification and response to therapy. Thromb Haemost 1999;81:705-10 http://www.ncbi.nlm.nih.gov/pubmed/ 10365741

24 Jing J, Zheng S, Han C, et al. Evaluating the value of uPAR of serum and tissue on patients with cervical cancer. J Clin Lab Anal 2012;26:16-21.

25 Riisbro R, Stephens RW, Brünner N, et al. Soluble urokinase plasminogen activator receptor in preoperatively obtained plasma from patients with gynecological cancer or benign gynecological diseases. Gynecol Oncol 2001;82:523-31.

26 Lomholt AF, Høyer-Hansen G, Nielsen HJ, et al. Intact and cleaved forms of the urokinase receptor enhance discrimination of cancer from non-malignant conditions in patients presenting with symptoms related to colorectal cancer. Br J Cancer 2009;101:992-7.

27 Usnarska-Zubkiewicz L, Strutyńska-Karpińska M, ZubkiewiczKucharska A, et al. Soluble urokinase-type plasminogen activator receptor and ferritin concentration in patients with advanced alimentary tract carcinoma. Relationship to localization, surgical treatment and the stage of the disease - preliminary report. Adv Clin Exp Med 2014;23:959-67.

28 Fidan E, Mentese A, Ozdemir F, et al. Diagnostic and prognostic significance of $\mathrm{Ca}$ IX and suPAR in gastric cancer. Med Oncol 2013;30:540.

29 Chounta A, Ellinas C, Tzanetakou V, et al. Serum soluble urokinase plasminogen activator receptor as a screening test for the early diagnosis of hepatocellular carcinoma. Liver Int 2015;35:601-7.

30 Rubio-Jurado B, Tello-González A, Bustamante-Chávez L, et al. Circulating levels of urokinase-type plasminogen activator receptor and D-dimer in patients with hematological malignancies. Clin Lymphoma Myeloma Leuk 2015;15:621-6.

31 Henic E, Borgfeldt C, Christensen IJ, et al. Cleaved forms of the urokinase plasminogen activator receptor in plasma have diagnostic potential and predict postoperative survival in patients with ovarian cancer. Clin Cancer Res 2008;14:5785-93.

32 Wach S, Al-Janabi O, Weigelt $\mathrm{K}$, et al. The combined serum levels of $m i R-375$ and urokinase plasminogen activator receptor are suggested as diagnostic and prognostic biomarkers in prostate cancer. Int J Cancer 2015;137:1406-16.

33 Cobos E, Jumper C, Lox C. Pretreatment determination of the serum urokinase plasminogen activator and its soluble receptor in advanced small-cell lung cancer or non-small-cell lung cancer. Clin Appl Thromb Hemost 2003;9:241-6.

34 Miyake H, Hara I, Yamanaka K, et al. Elevation of serum levels of urokinase-type plasminogen activator and its receptor is associated with disease progression and prognosis in patients with prostate cancer. Prostate 1999;39:123-9.

35 Rigolin GM, Tieghi A, Ciccone M, et al. Soluble urokinase-type plasminogen activator receptor (suPAR) as an independent factor 
predicting worse prognosis and extra-bone marrow involvement in multiple myeloma patients. Br J Haematol 2003;120:953-9.

36 Riisbro R, Christensen IJ, Piironen T, et al. Prognostic significance of soluble urokinase plasminogen activator receptor in serum and cytosol of tumor tissue from patients with primary breast cancer. Clin Cancer Res 2002;8:1132-41.

37 Enocsson H, Wetterö J, Skogh T, et al. Soluble urokinase plasminogen activator receptor levels reflect organ damage in systemic lupus erythematosus. Trans/ Res 2013;162:287-96.

38 Toldi G, Bekő G, Kádár G, et al. Soluble urokinase plasminogen activator receptor (suPAR) in the assessment of inflammatory activity of rheumatoid arthritis patients in remission. Clin Chem Lab Med 2013;51:327-32.

39 Portelli MA, Siedlinski M, Stewart CE, et al. Genome-wide protein QTL mapping identifies human plasma kallikrein as a post-translational regulator of serum UPAR levels. FASEB $j$. 2014;28:923-34.

40 Zimmermann HW, Koch A, Seidler S, et al. Circulating soluble urokinase plasminogen activator is elevated in patients with chronic liver disease, discriminates stage and aetiology of cirrhosis and predicts prognosis. Liver Int 2012;32:500-9.

41 Wiese S, Mortensen C, Gøtze JP, et al. Cardiac and proinflammatory markers predict prognosis in cirrhosis. Liver Int 2014:34:e19-30.

42 Sjöwall C, Martinsson K, Cardell K, et al. Soluble urokinase plasminogen activator receptor levels are associated with severity of fibrosis in nonalcoholic fatty liver disease. Trans/ Res 2015;165:658-66.

43 Meijers B, Poesen R, Claes K, et al. Soluble urokinase receptor is a biomarker of cardiovascular disease in chronic kidney disease. Kidney Int 2015;87:210-6.

44 Schaefer F, Trachtman H, Wühl E, et al. Association of serum soluble urokinase receptor levels with progression of kidney disease in children. JAMA Pediatr 2017;171:e172914.

45 Sevgi DY, Bayraktar B, Gündüz A, et al. Serum soluble urokinasetype plasminogen activator receptor and interferon- $\gamma$-induced protein 10 levels correlate with significant fibrosis in chronic hepatitis B. Wien Klin Wochenschr 2016;128:28-33.

46 Sidenius N, Sier CFM, Ullum H, et al. Serum level of soluble urokinase-type plasminogen activator receptor is a strong and independent predictor of survival in human immunodeficiency virus infection. Blood 2000;96:4091-5.

47 Kirkegaard-Klitbo DM, Langkilde A, Mejer N, et al. Soluble urokinase plasminogen activator receptor is a predictor of incident non-AIDS comorbidity and all-cause mortality in human immunodeficiency virus type 1 infection. $J$ Infect Dis 2017;216:819-23.

48 Hoenigl M, Raggam RB, Wagner J, et al. Diagnostic accuracy of soluble urokinase plasminogen activator receptor (suPAR) for prediction of bacteremia in patients with systemic inflammatory response syndrome. Clin Biochem 2013;46:225-9.

49 Wittenhagen P, Kronborg G, Weis N, et al. The plasma level of soluble urokinase receptor is elevated in patients with Streptococcus pneumoniae bacteraemia and predicts mortality. Clin Microbiol Infect 2004;10:409-15.

50 Donadello K, Scolletta S, Taccone FS, et al. Soluble urokinase-type plasminogen activator receptor as a prognostic biomarker in critically ill patients. J Crit Care 2014;29:144-9.

51 Koch A, Voigt S, Kruschinski C, et al. Circulating soluble urokinase plasminogen activator receptor is stably elevated during the first week of treatment in the intensive care unit and predicts mortality in critically ill patients. Crit Care 2011;15:R63.

52 Tzanakaki G, Paparoupa M, Kyprianou M, et al. Elevated soluble urokinase receptor values in CSF, age and bacterial meningitis infection are independent and additive risk factors of fatal outcome. Eur J Clin Microbiol Infect Dis 2012;31:1157-62.

53 Østergaard C, Benfield T, Lundgren JD, et al. Soluble urokinase receptor is elevated in cerebrospinal fluid from patients with purulent meningitis and is associated with fatal outcome. Scand $J$ Infect Dis 2004;36:14-19.

54 Wittenhagen P, Andersen JB, Hansen A, et al. Plasma soluble urokinase plasminogen activator receptor in children with urinary tract infection. Biomark Insights 2011;6:BMI.S6876.

55 Wrotek A, Jackowska T, Pawlik K. Soluble urokinase plasminogen activator receptor: an indicator of pneumonia severity in children. Adv Exp Med Biol 2015;835:1-7.

56 Savva A, Raftogiannis M, Baziaka F, et al. Soluble urokinase plasminogen activator receptor (suPAR) for assessment of disease severity in ventilator-associated pneumonia and sepsis. $J$ Infect 2011;63:344-50.

57 Rabna P, Andersen A, Wejse C, et al. Utility of the plasma level of suPAR in monitoring risk of mortality during TB treatment. PLoS One 2012; 7:e43933.

58 Perch M, Kofoed PE, Fischer TK, et al. Serum levels of soluble urokinase plasminogen activator receptor is associated with parasitemia in children with acute Plasmodium falciparum malaria infection. Parasite Immunol 2004;26:207-11.

59 Plewes K, Royakkers AA, Hanson J, et al. Correlation of biomarkers for parasite burden and immune activation with acute kidney injury in severe falciparum malaria. Malar J 2014;13:91

60 Andersen O, Eugen-Olsen J, Kofoed K, et al. Soluble urokinase plasminogen activator receptor is a marker of dysmetabolism in HIVinfected patients receiving highly active antiretroviral therapy. J Med Virol 2008;80:209-16.

61 Rasmussen LJH, Moffitt TE, Arseneault L, et al. Association of adverse experiences and exposure to violence in childhood and adolescence with inflammatory burden in young people. JAMA Pediatr 2019;4:1-11.

62 Rasmussen LJH, Moffitt TE, Eugen-Olsen J, et al. Cumulative childhood risk is associated with a new measure of chronic inflammation in adulthood. $J$ Child Psychol Psychiatry 2019;60:199-208.

63 Riley RD, Moons KGM, Snell KIE, et al. A guide to systematic review and meta-analysis of prognostic factor studies. BMJ 2019;6:k4597.

64 Shamseer L, Moher D, Clarke M, et al. Preferred reporting items for systematic review and meta-analysis protocols (PRISMA-P) 2015: elaboration and explanation. BMJ 2015;349:97647.

65 Moher D, Shamseer L, Clarke M, et al. Preferred reporting items for systematic review and meta-analysis protocols (PRISMA-P) 2015 statement. Syst Rev 2015;4:1.

66 Moher D, Liberati A, Tetzlaff $\mathrm{J}$, et al. Preferred reporting items for systematic reviews and meta-analyses: the PRISMA statement. BMJ 2009;339:b2535.

67 Stroup DF, Berlin JA, Morton SC, et al. Meta-Analysis of observational studies in epidemiology: a proposal for reporting. meta-analysis of observational studies in epidemiology (moose) group. JAMA 2000;283:2008-12.

68 Hayden JA, van der Windt DA, Cartwright JL, et al. Assessing bias in studies of prognostic factors. Ann Intern Med 2013;158:280-6.

69 Hemingway H, Philipson P, Chen R, et al. Evaluating the quality of research into a single prognostic biomarker: a systematic review and meta-analysis of 83 studies of C-reactive protein in stable coronary artery disease. PLoS Med 2010;7:e1000286.

70 Chêne G, Thompson SG. Methods for summarizing the risk associations of quantitative variables in epidemiologic studies in a consistent form. Am J Epidemiol 1996;144:610-21.

71 Higgins JPT, Thomas J, Chandler J, et al, eds. Cochrane Handbook for Systematic Reviews of Interventions version 6.0 (updated July 2019). Cochrane, 2019. www.training.cochrane.org/handbook

72 Guyatt GH, Oxman AD, Vist GE, et al. Grade: an emerging consensus on rating quality of evidence and strength of recommendations. BMJ 2008:336:924-6.

73 Huguet A, Hayden JA, Stinson J, et al. Judging the quality of evidence in reviews of prognostic factor research: adapting the grade framework. Syst Rev 2013;2:71.

74 Schultz M, Rasmussen LJH, Andersen MH, et al. Use of the prognostic biomarker suPAR in the emergency department improves risk stratification but has no effect on mortality: a cluster-randomized clinical trial (triage III). Scand J Trauma Resusc Emerg Med 2018;26:69.

75 Schultz M, Rasmussen LJH, Kallemose T, et al. Availability of suPAR in emergency departments may improve risk stratification: a secondary analysis of the triage III trial. Scand J Trauma Resusc Emerg Med 2019;27:43.

76 Botha S, Fourie CMT, Schutte R, et al. Soluble urokinase plasminogen activator receptor as a prognostic marker of all-cause and cardiovascular mortality in a black population. Int J Cardiol 2015;184:631-6.

77 Hayek SS, Sever S, Ko Y-A, et al. Soluble urokinase receptor and chronic kidney disease. N Engl J Med 2015;373:1916-25.

78 Wei C, El Hindi S, Li J, et al. Circulating urokinase receptor as a cause of focal segmental glomerulosclerosis. Nat Med 2011;17:952-60. 